\title{
Anesthetic Management of a Parturient with Thalidomide Phocomelia
}

\author{
Samuel N. Blacker, Tiffany E. Angelo \\ ${ }^{1}$ Department of Anesthesiology, Womack Army Medical Center, Fort Bragg, USA; ${ }^{2}$ Department of Anesthesiology, Walter Reed \\ National Military Medical Center, Bethesda, USA. \\ Email: Samuel.blacker@gmail.com
}

Received April 10 ${ }^{\text {th }}$, 2012; revised May 21 ${ }^{\text {st }}$ 2012; accepted June $18^{\text {th }}, 2012$

\begin{abstract}
The teratogenicity of thalidomide has been known since the early 1960s [1]. Thalidomide is currently used world wide, including the United States, to treat erythema nodosum leprosum, multiple myeloma, refractory Crohn's disease, aphthous stomatitis and HIV wasting syndrome. New cases of thalidomide phocomelia are being reported as well. We report a case of the anesthetic challenges of a 23 year-old parturient with thalidomide phocomelia and review the important anesthetic challenges it presents. Spontaneous vaginal delivery under continuous lumbar epidural was achieved in this challenging patient. However, it required careful planning for reliable intravenous access and the use of magnetic resonance imaging (MRI) of her pelvis and lumbar spine.
\end{abstract}

Keywords: Anesthesia; Thalidomide Phocomelia; Obstertric Anesthesia

\section{Introduction}

A 23 year-old G2 P0010 woman presented at 36 weeks gestation for an anesthetic consultation of the management of her four-limb phocomelia likely secondary to thalidomide exposure in utero. The patient was adopted from Thailand without any historical evidence of her biological parents' medical histories. Through multiple visits as a child to physicians and genetic counselors it was determined that her phocomelia was likely secondary to thalidomide exposure in utero. A plan for the patient to deliver vaginally was made after an MRI of the pelvis at 37 weeks gestation demonstrated adequate pelvic dimensions.

On physical examination the patient had four limb phocomelia. Her left upper extremity was 12 centimeter with a rudimentary wrist and one full finger. Here right upper extremity was 10 centimeters and blunt ended. The left and right lower extremities were 20 centimeters long with rudimentary ankle joints, with two toes on the left and three on the right. The left and right upper extremities lacked any physical structures consistent with an elbow joint or antecubital fossa and the lower extremities lacked knee joints. The patient's head, neck, and torso appeared grossly normal on exam. Her heart and lung exam were also unremarkable. The patient's spine was without evidence of scoliosis. The patient weighed 50 kilograms, was 114 centimeters tall and had a Malampati class II airway with three finger breadths thyromental distance. She also had full range of motion in her cervical spine. The previous MRI of the pelvis was re-examined by the radiologist and was found to be devoid of evidence of lumbar vertebral abnormalities. Her hematocrit was 33.9 and platelet count was 254. The patient also had a history of von Willebrand's disease but lacked antigen evidence upon her most recent testing.

The anesthetic plan consisted of non-invasive blood pressure monitoring via a standard blood pressure cuff on either upper extremity; pulse oximeter on the patient's one finger or via ear probe and standard electrocardiogram. These monitoring modalities were tested on our patient prior to labor and were deemed appropriate for peripartum use. Due to the patient's history of difficult intravenous access, lack of antecubital fossas, and the possibility of abnormal upper extremity vascular anatomy [2] a peripheral intravenous central catheter (PICC) was scheduled to be placed by interventional radiology at 37 weeks gestation. Right internal jugular intravenous access under ultrasound guidance was planned if the patient began laboring prior to her 37th wk gestation or if PICC placement was unsuccessful. Regional anesthesia would be used for labor analgesia with a secondary option of intravenous fentanyl via patient controlled analgesia pump. Should a cesarean section be necessary, standard neuraxial anesthetic with general anesthesia backup was planned.

The patient arrived in labor at 38 wks gestation. She 
had a functioning PICC line in place from the 37th wk gestation and an uncomplicated lumbar epidural (L3-4) was placed for labor analgesia at $4 \mathrm{~cm}$ dilation. Loss of resistance to air was at five centimeters $(\mathrm{cm})$ and the catheter was threaded easily five $\mathrm{cm}$ into the epidural space. The epidural catheter was incrementally bolused to $10 \mathrm{ml}$ of $0.125 \%$ bupivicaine and a T10 level was achieved bilaterally. A continous infusion of $0.125 \%$ bupivicaine with 2 micrograms (mcg) per milliliter (ml) of fentanyl was started at that time at $8 \mathrm{ml}$ per hour with blousing option of an additional $3 \mathrm{ml}$ every 20 minutes. The patient labored for eleven hours under epidural analgesia, which resulted in an uncomplicated vaginal delivery of a healthy baby boy. The epidural was turned off one hour after her delivery and removed 3 hours later. The patient's post-partum course was uneventful and she was discharged home on post-partum day 3. The PICC line was removed prior to her hospital discharge.

\section{Discussion}

Our case demonstrates the importance of a working knowledge of the potential anesthetic difficulties encountered in patients with thalidomide phocomelia. The potential teratogenicity of thalidomide was first described in Germany and Australia in 1961 [3]. At that time thalidomide was used as an antiemetic. Its use continues in the United States, as thalidomide was approved by the Food and Drug Administration in 1998 for the treatment of erythema nodosum leprosum [4]. The fetal abnormalities are attributed to the medication's anti-angiogenic properties. This effect is most notably recognized in the inhibition of the formation of blood vessels in newly forming limbs [5]. This inhibition leads to the classical physical findings of phocomelia (the absence of proximal elements of the limb) or amelia (the complete absence of a limb). In addition to deleterious effects on the limbs, thalidomide has also been associated with anterior fusions of the lower thoracic and lumbar vertebrae, spina bifida, scoliosis, congenital heart defects, cranial nerve defects, tear production abnormalities, depression of the nasal bridge, and deafness. It has also been shown to cause malformations of the reproductive and urologic systems as well as deformities of the pelvis and sacrum [6]. The first consideration when evaluating this patient was the patient's reduced total blood volume. Her extremity anatomy and reduced skeletal muscle mass reduces the vascular reservoir. This increased the likelihood of transfusion and further necessitated early and reliable IV access prior to labor. Communication with the blood bank was paramount.

Successful general anesthesia has been performed in these patients with observed faster inductions, emergences, and responses to titration of medications. This has been attributed to the reduction in body skeletal muscle mass and associated vessels [7]. Our patient's external airway anatomy was normal by exam. We did not pursue neck MRI or bronchoscopy to further evaluate her airway anatomy, as she had no known midline defects and previous case reports do not report difficulty with intubation in these patients [8].

In the parturient with history of thalidomide exposure however, there is increased risk to the patient and fetus because of potential lumbar and pelvic anatomical abnormalities. Standard history and physical examination do not reveal the full extent of thalidomide patient's anatomical abnormalities. MRI offers invaluable information on the presence and extent of defects of the pelvis and spinal anatomy to determine feasibility and safety of regional anesthesia. MRI also provides pelvic dimensions and anatomy to determine safety and feasibility of vaginal delivery. Without known pelvic outlet dimensions and lumbar spine anatomy, a cesarean section under general anesthesia would be the delivery method of choice, as reported with previous similar patients [9]. Therefore a well functioning epidural was vital for an attempt at a vaginal delivery.

Our patient demonstrated no abnormalities that prevented epidural placement such as fusions or severe scoliosis. The epidural placement was uncomplicated and we noted no change in the blood pressure with titration of the epidural bupivicaine to a level of T10. This may be due to slow, careful titration, but the lower vascular volume of distribution is likely a factor. We encountered no difficulty with the epidural, but without MRI we would not have been able to rule out vertebral fusions preventing epidural placement.

Had the MRI revealed unfavorable pelvic anatomy, but normal vertebral anatomy, a spinal anesthesic for cesarean section would have been our chosen technique. We thought a normal spinal anesthetic dose would be appropriate with normal vertebral anatomy, including the height and shape, that was consistent with the torso of a normal adult female. Therefore, the limiting factors for the achieved level of a spinal anesthetic would remain the same as it would for a regular patient.

Anesthesiologists in any country can anticipate encountering thalidomide patients, as we did, because of continued worldwide use and because of adoption, immigration, and patients seeking care in other countries. Thalidomide, although no longer used in pregnancy, is still used to treat select groups of patients. Off label uses include multiple myeloma, refractory Crohn's disease, aphthous stomatitis and HIV wasting syndrome [10]. Future phocomelia may not only be caused by maternal use, as thalidomide has been measured in seminal fluid of patients on the medication [11]. Threshold dose for teratogenicity is not known and any exposure is a risk. 
Given the continued thalidomide use, thalidomide embryopathy continues to be clinically relevant and likely so in the future, as demonstrated by our case and new cases reported in Brazil [12].

In conclusion, we present a case of a parturient with thalidomide-induced phocomelia. In view of the potential systemic abnormalities seen in thalidomide exposure, early consultation and discussion of analgesic and anesthetic options should be reviewed with parturients and their providers. Further testing outside of a physical exam that may prove to be beneficial includes: EKG, echocardiogram and MRI of the lumbar spine and pelvis. Early planning for vascular access, pulse oximetry and blood pressure monitoring is also recommended.

\section{Disclaimer}

The views expressed in this article are those of the authors and do not necessarily reflect the official policy or position of the Department of the Army, Navy, Department of Defense, nor the U.S. Government.

\section{REFERENCES}

[1] W. G. McBride, "Thalidomide and Congenital Abnormalities (Letter),” Lancet, Vol. 1, 1962, p. 45.

[2] P. J. Barrett and J. F. McGeachie, "Anaesthesia and Thalidomide-Related Abnormalities,” International Journal of Obstetric Anesthesia, Vol. 1, No. 4, 1992, pp. 235-236. doi:10.1016/0959-289X(92)80012-H

[3] W. Lenz, "Thalidomide and Congenital Abnormalities (Letter),” Lancet, Vol. 2, 1961, p. 1358.

[4] S. L. Nightingale, "From the Food and Drug Administration: Thalidomide Approved for Erythema Nodosum Leprosum,” Journal of the American Medical Association, Vol. 280, 1998, p. 872. doi:10.1001/jama.280.10.872

[5] C. Therapontos, L. Erskine, E. R. Gardner, F. D. William, N. Vargesson, “Thalidomide Induces Limb Defects by Preventing Angiogenic Outgrowth during Early Limb Formation," Proceedings of the National Academy of Sciences of the United States of America, Vol. 106, No. 21, 2009, pp. 8573-8578. doi:10.1073/pnas.0901505106

[6] C. G. H. Newman, "Clinical Observations on the Thalidomide Syndrome," Journal of the Royal Society of Medicine, Vol. 70, No. 4, 1977, pp. 225-227.

[7] J. W. McCrory, "Anaesthesia and Thaloidomide-Related Abnormalities,” Anaesthesia, Vol. 43, No. 7, 1988, pp. 613-614. doi:10.1111/j.1365-2044.1988.tb06736.x

[8] A. R. Williams and M. K. Bailey, "Anesthetic Management of a Patient with Tetra-Amelia,” Southern Medical Journa, Vol. 92, No. 3, 1999, pp. 325-327. doi:10.1097/00007611-199903000-00014

[9] P. G. Maouris and P. J. Hirsch, "Pregnancy in Women with Thalidomide-Induced Disabilities. Case Report and a Questionnaire Study,” British Journal of Obstetrics and Gynaecology, Vol. 95, No. 7, 1988, pp. 717-719. doi:10.1111/j.1471-0528.1988.tb06536.x

[10] R. J. Powell. “Thalidomide: Current Uses,” BioDrugs, Vol. 11, No. 6, 1999, pp. 409-416. doi:10.2165/00063030-199911060-00005

[11] S. K. Teo, J. L. Harden, A. B. Burke, F. H. Noormohamed, M. Youle, M. A. Johnson, B. S. Peters, D. I. Stirling and S. D. Thomas, "Thalidomide Is Distributed into Human Semen after Oral Dosing,” Drug Metabolism and Disposition, Vol. 29, No. 10. 2001, pp. 1355-1357.

[12] L. Schuler-Fuccini, R. C. Soares, A. C. de Sousa, C. Maximino, E. Luna, I. V. Schwartz, C. Waldman and E. E. Castilla, "New Cases of Thalidomide Embryopathy in Brazil,” Birth Defects Research, Vol. 79, No. 9, 2007, pp. 671-672. doi:10.1002/bdra.20384 